Published in International Journal of Music Education, 2019, vol. 37, no. 2, pp. 311-326, , which should be cited to refer to this work. https://doi.org/10.1177/0255761419827342

RUNNING HEAD: INFLUENCE OF BODY AWARENESS ON INSTRUMENTAL SOUND

\title{
Exploring the influence of body awareness on instrumental sound
}

Claudia Dora, Simone Conforti and Angelika Güsewell

Author Note

Claudia Dora, University of Applied Sciences and Arts Western Switzerland (HES-SO), Haute Ecole de Musique de Genève, Site de Neuchâtel, Switzerland;

Simone Conforti, Universität Basel, Switzerland;

Angelika Güsewell, University of Applied Sciences and Arts Western Switzerland (HES-SO), Haute Ecole de Musique Vaud Valais Fribourg, Switzerland.

\begin{abstract}
Results from recent research have demonstrated positive effects of somatic approaches, such as the Feldenkrais method, somasthetics, and body mapping in the field of music. However, the direct impact of such approaches on instrumental sound was never studied so far. The present pilot study was thus designed to investigate the influence of non-judgmental body awareness on the sound of high string instruments. $\mathrm{N}=11$ students of the music colleges of Geneva and Lausanne were requested to sense specific parts of their body while playing one long note. The analysis of audio recordings and interviews conducted post hoc show a positive correlation between the subjective experience of ease participants reported, and objective sound volume or stability. The largest effects were observed when participants were aware of their pelvis, rib cage or head region. Increases of sound volume or stability were often accompanied by non-voluntary changes in body weight distribution, indicating that body awareness had a direct influence on the musculoskeletal system. In view of the reportedly high numbers of playing-related health problems in professional orchestra players, more research should be carried out to examine in detail possible effects of body awareness on instrumental sound and on psychological and physical well-being.
\end{abstract}

Keywords: Body awareness, instrumental sound, mindfulness, music, sound analysis 


\section{Introduction}

For any movement that has to do with transformation, we have to engage in direct bodily experience. The only thing we can do is to find our orientation in our organism, in processes. (Gindler, 1946, as cited in Ludwig, 2002, p. 150)

\section{Music and Somatic Approaches}

Recent qualitative studies about the influence of body work such as the Feldenkrais method, somaesthetics, body mapping, and other kinds of physiological approaches (Buchanan \& Hays, 2014; Paparo, 2015) demonstrate the potential of somatic approaches in the field of music. These studies suggest that bodywork leads to higher levels of body awareness, increased bodily presence, and musical expression for both singers and instrumentalists. Somatic approaches such as the Alexander technique, the Feldenkrais method, tai chi, and yoga have been established as a part of the curriculum at music colleges since the last decades of the 20th century, but the actual transfer of such approaches to the instrument seems to be difficult. Musicians not only invest a lot of individual training time to achieve a minimal level of acceptance in their professional field, but they continuously must prove their skills on stage and therefore remain subject to almost constant control by their colleagues and the audience. In such a competitive environment, the process of self-discovery and the incorporation of somatic work into individual instrumental technique can easily be regarded as a loss of time.

Constant stress, performance anxiety, and perfectionism, however, can trigger health problems typical for musicians, namely chronic pain and focal dystonia (Jäncke, 2011). Numerous studies document the high rate of playing-related, musculoskeletal problems among professional orchestra musicians (Gembris, Heye, \& Seifert, 2018; Leaver, Harris, \& Palmer, 2011; Steinmetz, Scheffer, Esmer, Delank, \& Peroz, 2015). Somatic approaches could play an essential role in the prevention of such problems. 
One key element of somatic work is body awareness (Mehling et al., 2011). As opposed to intentional, goal-oriented body control aimed at accomplishing a task in an efficient manner, body awareness, as defined here, is characterized by an attitude free of judgment. It includes 'a quality of relating to one's experience with an orientation of curiosity, experimental openness and acceptance' (Bishop et al., 2004, p. 234). The attentional focus is on the individual's being rather than on its doing.

\section{Body Awareness in the Work of Gindler and Jacoby}

One of the most influential Western pioneers exploring body awareness was the German movement teacher Elsa Gindler, whose work had a sustained impact in the fields of somatic psychology and body-oriented psychotherapy (Weaver, 2015). In the framework of female 'reform gymnastics' in Berlin, in the early 1920s she already had started experiments on sensing the body in 'the present moment'. Initially, she concentrated on the awareness of respiration or the experience of gravity in basic situations, such as sitting on a chair or lying on the ground. As of 1925, she continuously developed her working method in close collaboration with pianist and composer Heinrich Jacoby. Together with their students, this pair explored possibilities for overcoming hindering habits by seeking appropriate questions and tasks, leading to a spirit of exploration and discovery during direct self-experience.

In some of these experiments, initially called Tastversuche, participants were encouraged to sense regions of their bodies, e.g. the left foot, the left lower leg, the distance between left knee and hipbone, or the left arm as a whole. Gindler's long-term student and collaborator Sophie Ludwig (2002) notes that after these experiments, participants regularly reported that their muscles felt more 'naturally' heavy, that the corresponding body regions were energized, and that blood supply was increased. At the same time, body movements regularly felt 'surprisingly' light (Ludwig 2002, pp. 146-147).

Gindler and Jacoby were convinced that the human organism latently keeps its ability to function as a whole, even if it is used inadequately over long periods of time. In their opinion, 
step-by-step regeneration can be achieved by conscious bodily experience and the ability to differentiate forced action from organic action. Gindler and Jacoby did not consider their approach to be therapy but rather a possibility for adults to unfold their dormant potential; they called it simply 'the work', without creating a theory around it.

\section{Mindfulness-based Stress Reduction}

Body awareness is also a central element of mindfulness-based stress reduction (MBSR). Jon Kabat-Zinn, professor of medicine emeritus at the University of Massachusetts Medical School, developed the MBSR program. He has been engaged in introducing and spreading knowledge about Buddhist mindfulness practices in Western society and medicine. Kabat-Zinn (2012, p. 17-18) defines mindfulness as 'what arises when you pay attention on purpose, in the present moment, non-judgmentally, and as if your life depended on it'. He speaks of a 'being mode' as opposed to a 'doing mode' of mind, and that the shift from one mode to the other can be achieved by attention and awareness.

During the past two decades, mindfulness has become a common object of research in the fields of medicine, neuroscience, psychology, and education. Studies have been concerned with chronic pain (Zautra et al., 2008), depression and recovery from it (Teasdale et al., 2000), psychosomatic symptoms and emotional expression (Landsman-Dijkstra, Wijck, Groothoff, \& Rispens, 2004), brain activity (Kerr, Sacchet, Lazar, Moore, \& Jones, 2013; Tomasino \& Fabbro, 2016), attention deficient hyperactivity disorder (Zylowska et al., 2007), skill-acquisition and performance in sports (Kee \& John Wang, 2008; Zhang et al., 2016), stress management for medical and mathematics students as well as performance under challenging conditions (Bellinger, DeCaro, \& Ralston, 2015; Rosenzweig, Reibel, Greeson, Brainard, \& Hojat, 2003), and education in general (Bai, Beatch, Chang, \& Cohen, 2017).

In the field of music, mindfulness research has focused on musical performance and performance anxiety in the frame of mindfulness-based meditation (Lin, Chang, Zemon, \& Midlarsky, 2008), the development of psychological skills (Steyn, Steyn, Maree, \& 
Panebianco-Warrens, 2016), and vocal technique (Czajkowski \& Greasley, 2015). RodríguezCarvajal and Lecuona (2014) highlight music as a promising subject for further mindfulness research and, inter alia, point out the lack of empirical studies in this field.

One of the basic practices in the 8-week MBSR program is the body scan, an attention-focusing practice. It shows remarkable similarities to Gindler's Tastversuche. In a guided meditation, participants are directed to be aware of various parts of their body while sitting or lying in a comfortable position. Observations made by the participants can be at the physical, emotional, or cognitive level. As a result, the body scan offers the possibility to cultivate a distinction of the various bodily sensations. It helps to sustain non-judgmental attention and allows the perception of automatic judgments during experience. The body scan does not emphasize immediate or future change but the acceptance of whatever is in the focus of awareness, whether positive, negative, or neutral. It has received little attention as a standalone practice in mindfulness research (Dreeben, Mamberg, \& Salmon, 2013), although it is a central element of the MBSR program. A recent study suggests that in a clinical setting a brief mindfulness-based body scan has an immediate positive effect for people experiencing chronic pain (Ussher et al., 2014).

\section{Open Questions and Aims of this Research}

To date, mindfulness research in the field of music has tended to focus on mindfulness separate from action rather than mindfulness during action. Very little is known therefore about a possible direct influence of non-judgmental, plain body awareness during musical performance. This lack of research is surprising because the body is involved at any moment during musical action, be it practicing, teaching, rehearsing, or performing. The present research aims at contributing towards closure of this gap by exploring the relationship between body awareness and instrumental sound during musical activity. This leads to the following research questions:

1) Are there changes in sound when musicians maintain non-judgmental body 
awareness while playing?

2) What do participants experience when maintaining awareness of their body while playing?

3) Is there a relationship between the perceived experiences of participants and measured results?

\section{Methods}

To address these questions, three 'body journeys ${ }^{11}$ similar to the body scan and following a predetermined route along the body were used. Two types of data related to these journeys were collected: recordings of the long notes for sound analysis (i.e. quantitative data) and interviews in which participants shared their subjective experience during the body journeys (i.e. qualitative data).

\section{Participants}

Nine women and two men, age 18-25 $(M=22)$, took part in this research. All were studying at the music colleges of XXX or XXX, and all played a high string instrument, namely the violin $(n=10)$ or the viola $(n=1)$. Five volunteers were studying at the bachelor level and six at the master level. String players were chosen because it is possible for them to play continuous sounds that can be carried on independently from the breath.

\section{Procedure}

Body journeys. Participants were introduced approximately 20 minutes before the three body journeys (BJs). They were told to choose on their own a long note to play during the BJs with the sole condition that it should have little natural resonance (e.g. F, B, or C\#) to reduce the sympathetic vibrations of open strings to a minimum. The focus of each BJ was on a different body region. In BJ1, participants started by focusing their awareness on the left foot, shifting it after a moment to the left ankle, left knee, left hip joint, sacral bone, right hip

\footnotetext{
${ }^{1}$ Originally developed by one of us (C.D.) in 2001 in the context of workshops with professional string players to search for new sound qualities and feelings of greater ease while playing.
} 
joint, right knee, right ankle, and right foot. In BJ2, they started by focusing their awareness on the left finger that pressed down the string, shifting it after a while to the left hand, left wrist joint, left elbow, left shoulder, left shoulder blade, spine, whole rib cage, right shoulder blade, right shoulder, right elbow, right wrist joint, right hand, and right fingers. In BJ3, they started by focusing their awareness at the space above the head, shifting it after a while to the crane, down the spine to the sacrum and simultaneously down the back of their legs to the feet. Figure 1 displays the three body journeys ${ }^{2}$.

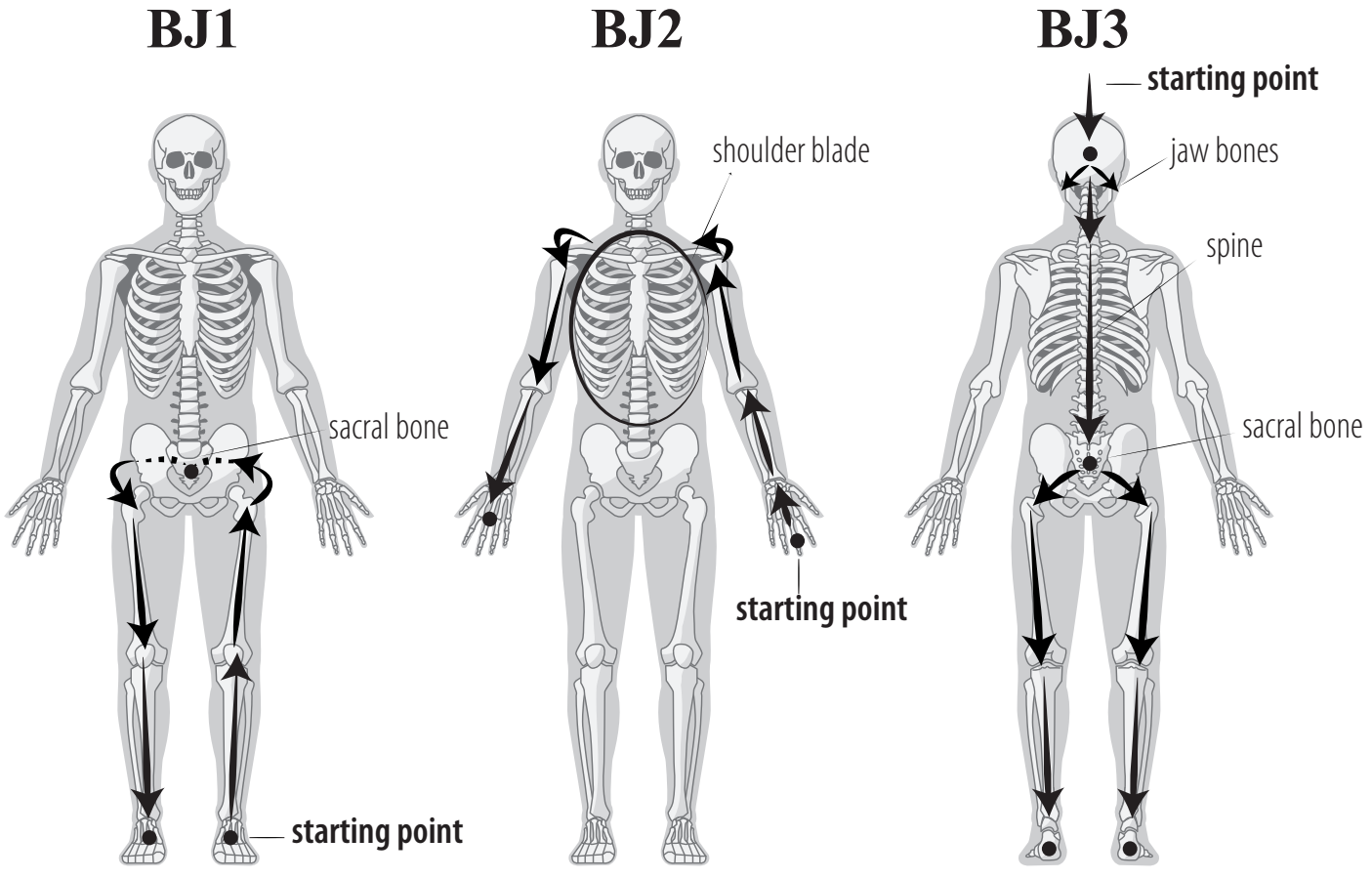

Figure 1. Three body journeys.

Participants were told to maintain body awareness as constant as possible during their body journeys. They also were encouraged to strive throughout for a benevolent attitude towards themselves, registering the fluctuation of body sensations from moment to moment.

\footnotetext{
${ }^{2}$ The special focus on feet and hands at the beginning and/or end of the three journeys is worth mentioning. From a body therapeutic view, hands and feet support grounding, i.e. ground contact and stability, which is relevant for any kind of work with the body. In journeys 1 and 2 we proposed to start at the left side of the body, although it would be fine to start on the right one should this feel better or seem easier.
} 
Finally, they were explicitly allowed to change the experiment spontaneously should they feel the need to do so (e.g. to change the course of their 'journey').

Audio recordings. The recordings of the long notes played during the BJs were settled with the aim of replicability across all journeys. Researchers took care of the maximal similarity concerning the equipment involved (i.e. microphone position, microphone preamplifier gain level, recording position).

Markers for important moments. To dispose of a time reference on which to rely during data analysis, participants were asked to give the researchers a sign whenever they felt that something special was happening that they might want to share during the interview, which would take place directly after the respective BJ. Such signs were given via eye contact (e.g. a wink) and allowed the sound engineer to set a marker as a point of orientation on the recording software.

Interviews. Short semi-structured interviews were conducted with participants after each BJ. First, participants were asked to explain with which body part the time markers corresponded and what had occurred at these points. They then were encouraged to mention any experience they wished to share. Finally, if not mentioned spontaneously, they were asked if they had noticed any change in sound (i.e. quality or volume) or in the fluidity of their movements. If they reported an increase in volume, they were asked at which point of their BJ it had occurred and if they felt they had used more physical effort during that moment. Notes taken during the interviews were transcribed and double-checked with the participants afterwards.

\section{Data Analysis}

Sound analysis. In a first step, the recordings from the BJs were analysed to explore if the sound had changed during one or more BJs, and if so, how and at what point. The 
psychoacoustical methods ${ }^{3}$ used to investigate the recordings were based on measurements of the spectral energy distribution as well as on the models of three psycho-acoustic descriptors: loudness (i.e. perceived volume), spectral centroid, and spectral roll-off (Peeters, 2004).

Different audio analysis software was tested for suitability for the purpose of this study; finally, AudioSculpt (version 3.4.5; IRCAM, 2016) was chosen.

Spectral energy measurement. The distribution of the spectral energy is defined by the dynamic level at which each partial of a sound is identifiable. This descriptor provides a clear visual representation of how the changes in timbre around the markers' zones are related to the different moments of each BJ. To read the data corresponding to spectral analysis correctly, we opted for a representation of the energy spectrum by means of different colours to clarify the dynamic ratio among the partials (Figure 2). The colour changes along the time axis display the sound transformations as they happen during a specific BJ and give a first impression of the spectral energy distribution.
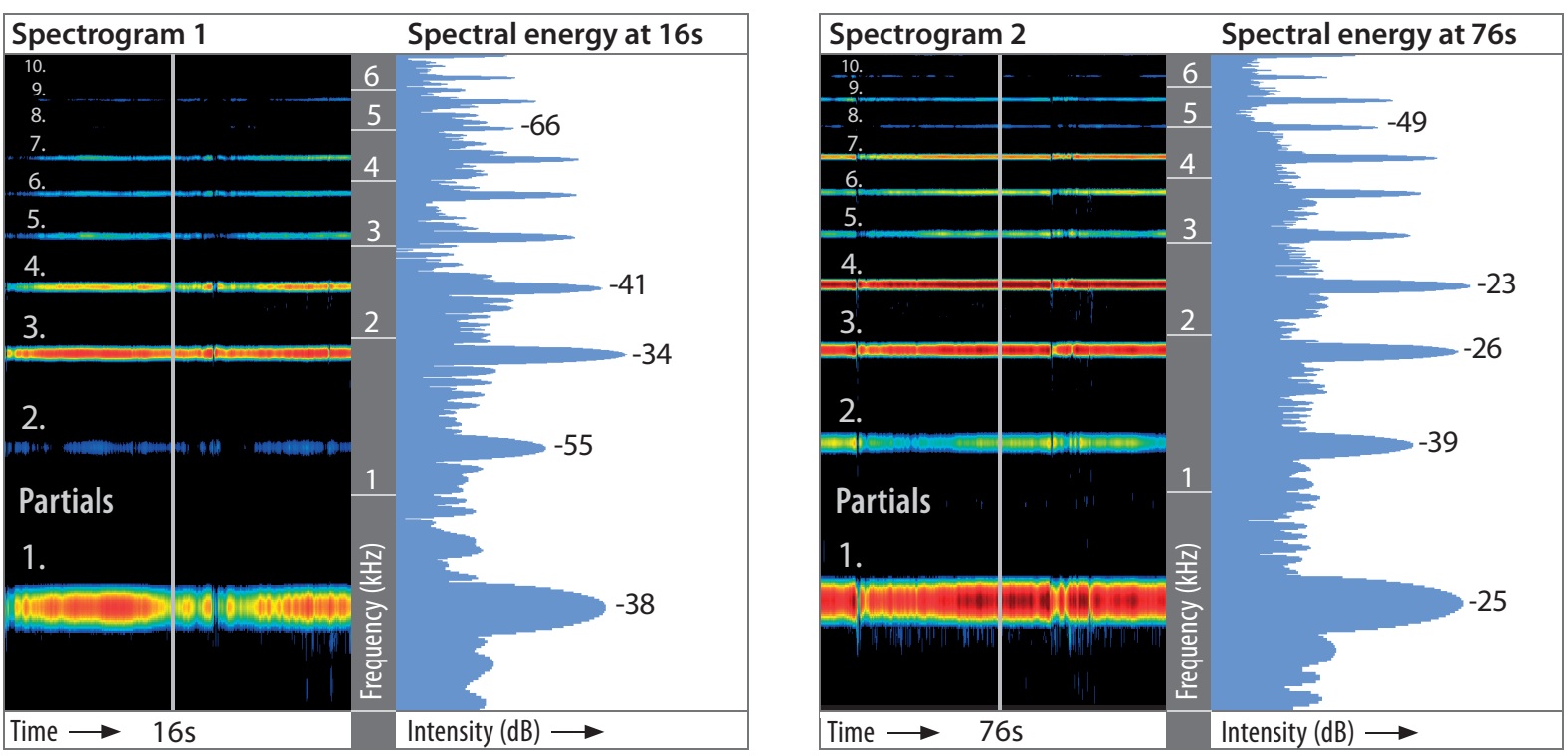

Figure 2. The left side of each picture shows the colour representation of the spectral energy distribution with the first 10 partials. Starting from black as the weakest sound representation

\footnotetext{
${ }^{3}$ Psychoacoustical research aims to establish quantitative relationships between the physical properties of a sound (i.e. the properties measured by the methods and instruments of the natural sciences) and the perceived properties of the sounds, the auditory attributes (Susini, Lemaitre, \& McAdams, 2012).
} 
of the FFT analysis, the plot changes over to blue, green, and yellow to red. The right side of each picture shows the $\mathrm{dB}$ measurement of each partial.

Loudness measurement. 'Loudness' is a psycho-acoustical rather than a physical attribute of sound. It is a measurement of the dynamic based on the human perception of the sound wave energy. It corresponds to the sound intensity and the spectral content of the sound signal. Whereas the sound intensity as well as the spectral content can be physically measured, the perception of loudness is a psychological quantity and is determined by the human listener. The loudness plot provides fundamental information to assess the impact of the experiment at the dynamic level. The sone measurement describes changes in volume based on the isophonic curves (i.e. equal-loudness contours). The display of the loudness (Figure 3) shows the graphic of the spectrum in the background as well. When a specific differentiation of the spectral energy distribution is observed, it likely corresponds to a change in loudness. In fact, sound changes can be objectified more deeply when a strict correlation between the spectral representation and the volume information (loudness) can be established.

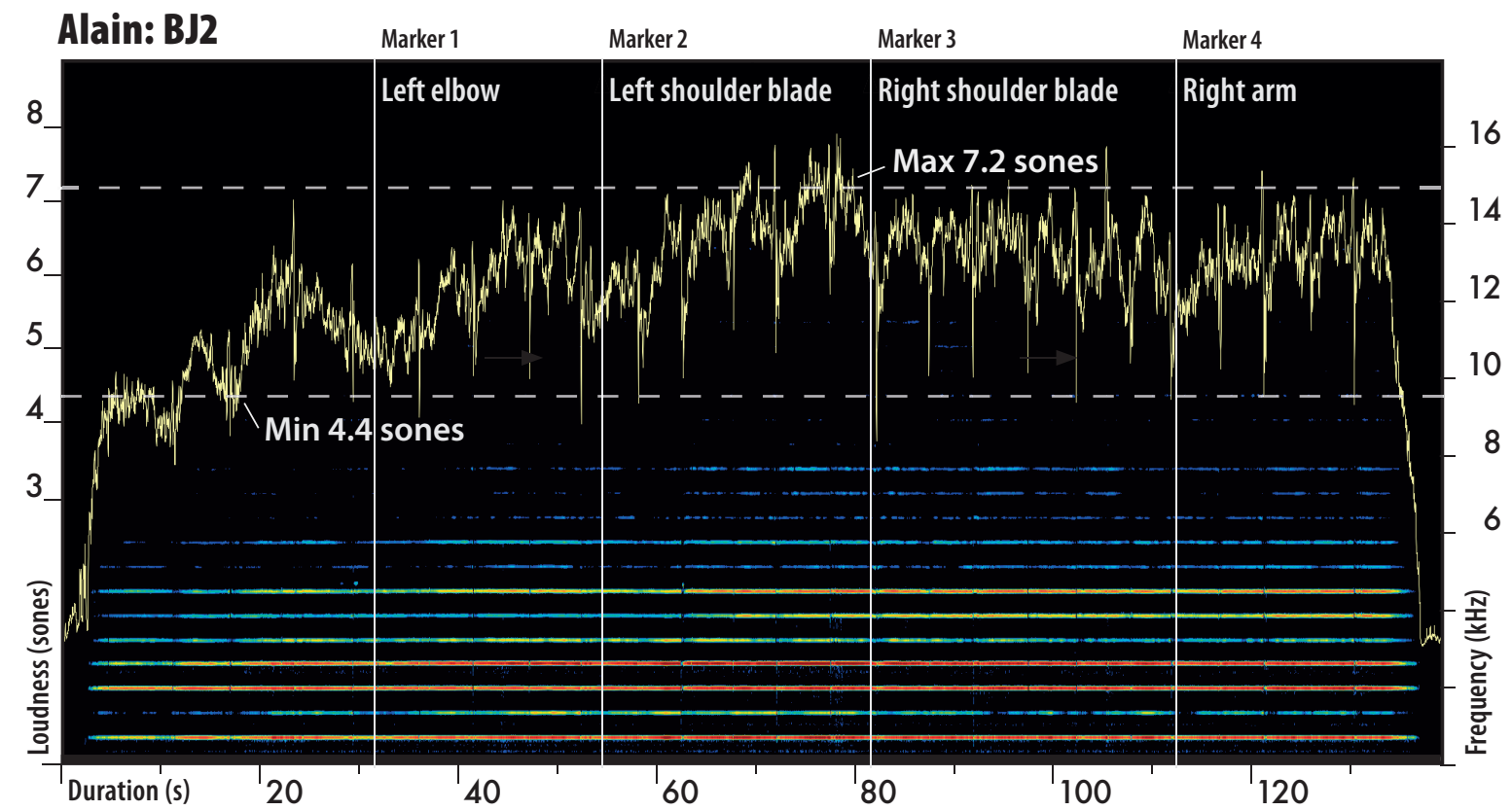

Figure 3. E flat ${ }^{2}$ on A string; spectrum shown in the background and loudness graph in yellow. The $\mathrm{X}$ axis represents the duration in seconds, the left $\mathrm{Y}$ axis the loudness scale in 
sone, and the right $\mathrm{Y}$ axis the frequency in $\mathrm{kHz}$. The loudness and spectral analysis show a clear increase of the sound's stability (dynamic and spectrum) in the second half of the experiment. Despite the continuous rise in the dynamics in the first half of the experiment, the ratio between the main partials is preserved on average from the beginning throughout the $\mathrm{BJ}$, even if a major number of higher partials is visible in the second half.

Spectral centroid. The spectral centroid is an audio descriptor that defines the perceived colour character of a sound (Figure 4). This descriptor indicates where the 'centre of mass' of the spectrum is located and helps one to understand if the main energy distribution of the different partials results in a 'darker' or 'brighter' sound. The spectral centroid's relative changes were used to measure the sound's barycentre to objectify the timbres described by participants.

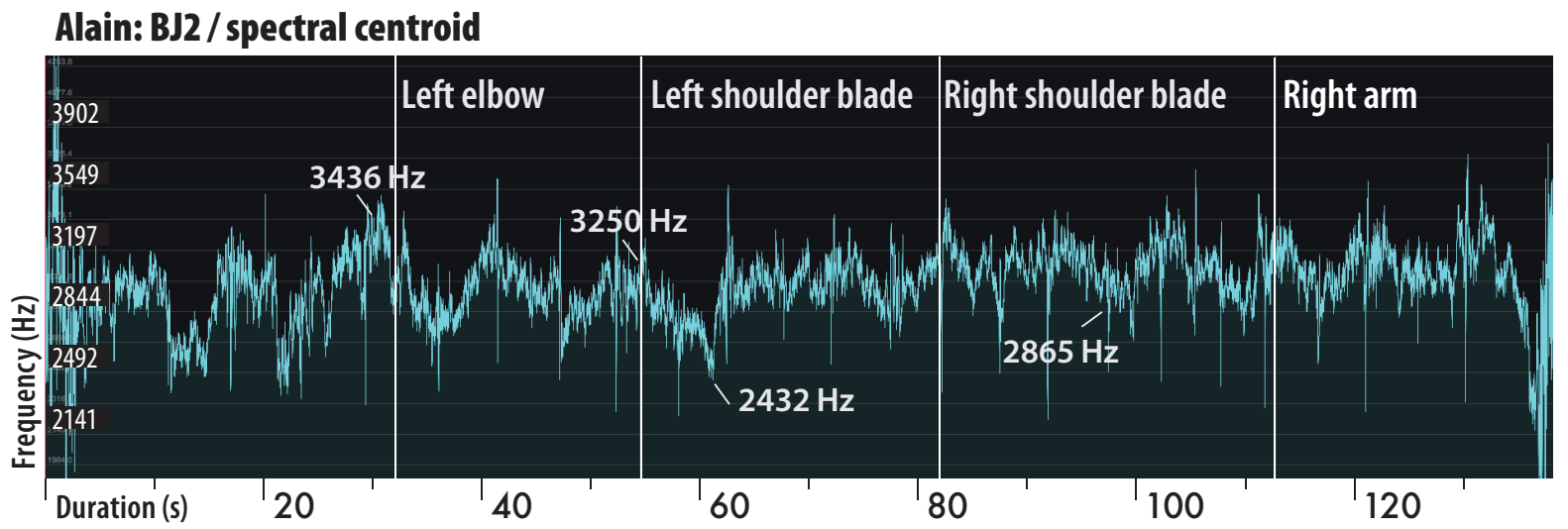

Figure 4. E flat ${ }^{2}$ on A string; spectral centroid graph shown in blue. The $\mathrm{X}$ axis represents the duration in seconds and the $\mathrm{Y}$ axis the spectral centroid in Hz. Note that while the loudness increases (see yellow curve in Figure 3) and the sound tends to have a wider spectrum, the centroid's frequency tends to decrease mostly near the marker's points.

Spectral roll-off. The spectral roll-off measurement defines the frequency margin above which the spectral energy is considerably weaker and thus can be considered less influential concerning the overall quality of the sound (Figure 5). This descriptor has been 
used as a guideline to support and delimit the analytical choices. In fact, the most relevant aspect is to relate to an objective measurement of the meaningfully hearable threshold that allows to stop the spectral investigation.

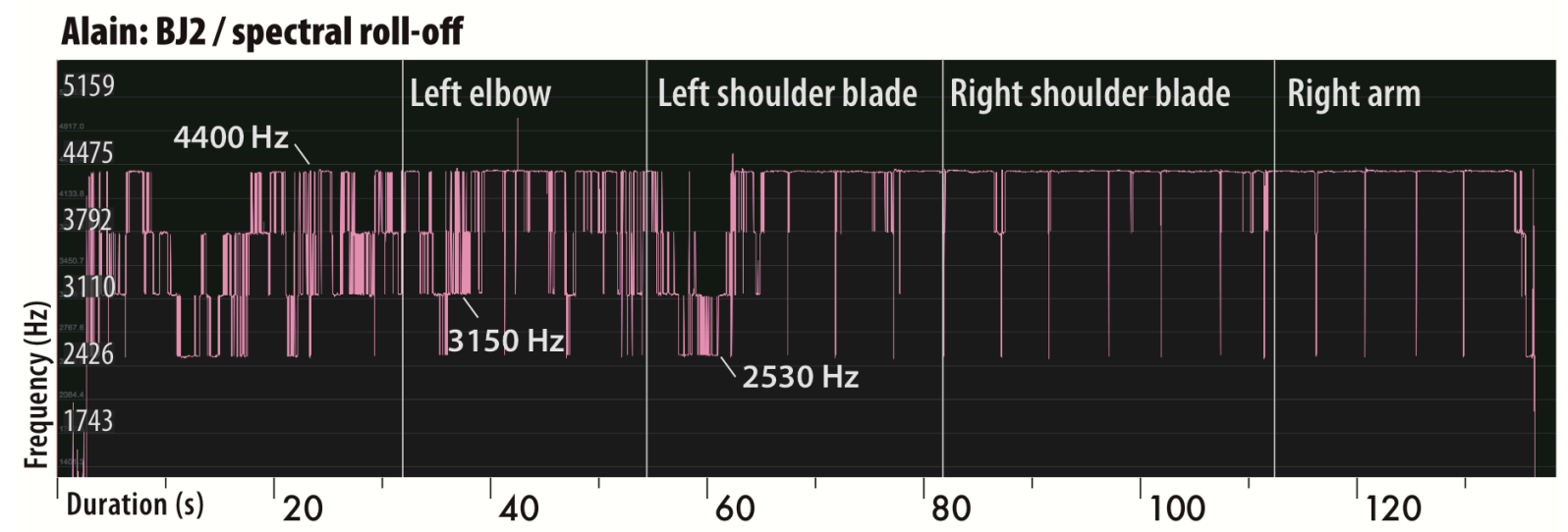

Figure 5. E flat ${ }^{2}$ on A string; spectral roll-off graph shown in purple. The $\mathrm{X}$ axis represents the duration in seconds and the Y axis the spectral roll-off in Hz. This example shows how the first half of the recording was characterized by a continuous fluctuation as opposed to the second half, which remained stable.

Comparing sound analysis and interview data. To explore how qualitative and quantitative data could be merged and a potential relationship between the participants' experiences and measurable results could be established, the sound analyses were compared with the notes from the interviews. The large amount of data was difficult to prioritize at first. After initial comparison of the data from recordings, sound analysis, and interviews, contrasting recordings of BJ2 (Alain, Michelle, Hanna) ${ }^{4}$ were selected. These participants' interviews were compared in detail with the corresponding spectral analysis, loudness, spectral centroid, and spectral roll-off. Figure 6 illustrates how interview data were compared with the spectral energy graph and loudness descriptor.

\footnotetext{
${ }^{4}$ Alain's BJ2 was characterized by slow, gradual changes, whereas Michelle shifted quite rapidly from one joint to the next. Hanna mentioned several times after the BJs that she was not able to sense her joints easily. She generally set only few markers and her BJ2 did not seem to have any particular structure or direction. (For video recordings of the three participants' BJ2 in comparison with sound analysis, see supplementary files.)
} 


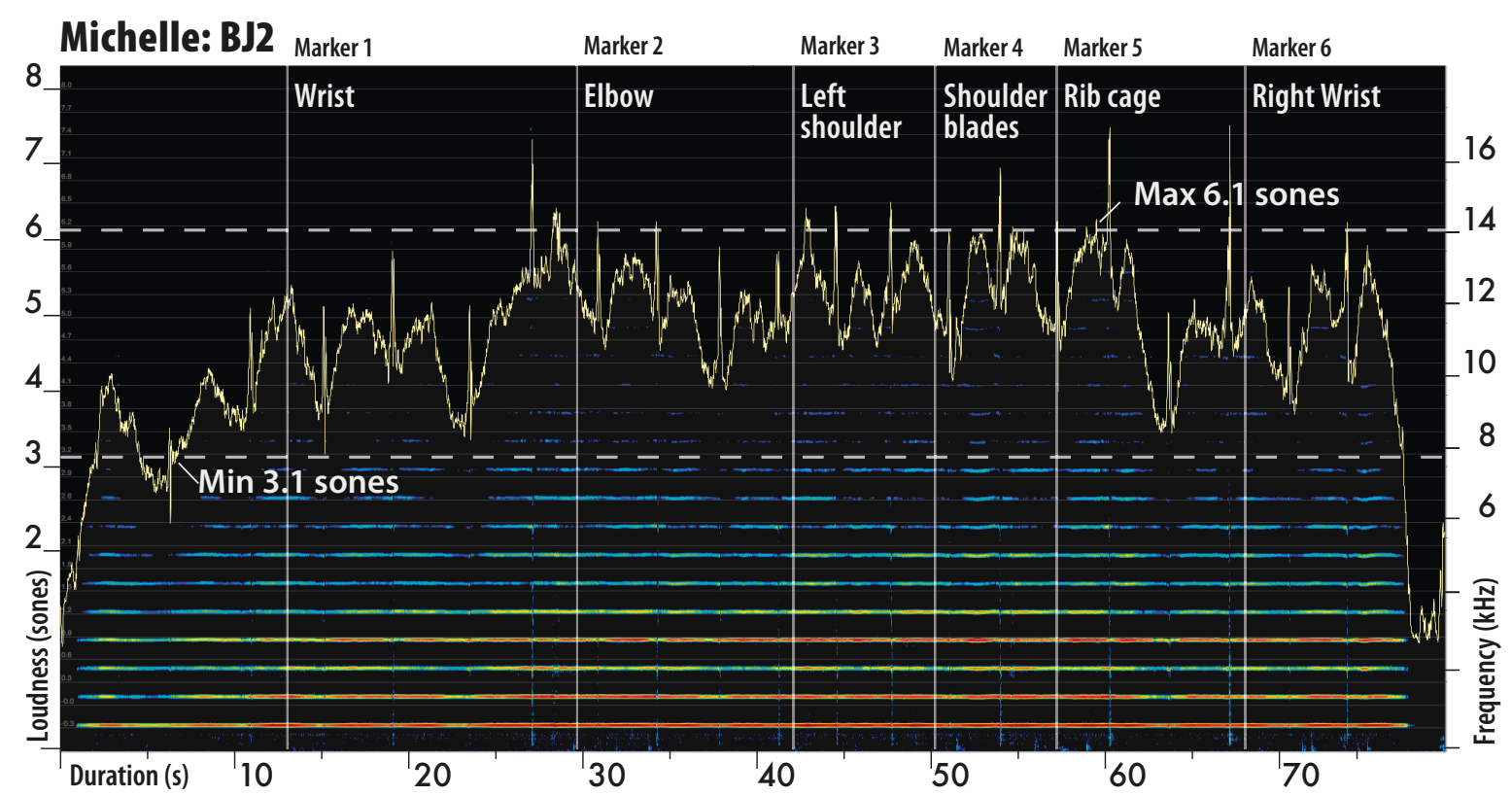

Figure 6. $\mathrm{G}^{2} /$ A string spectrum shown in the background and loudness graph shown in yellow. The $\mathrm{X}$ axis represents the duration in seconds, the left $\mathrm{Y}$ axis the loudness in sone, and the right $\mathrm{Y}$ axis the frequency in Hz. Michelle's BJ2 was characterized by a progressive enlargement of timbre and increase of volume. However, this was not achieved in a linear manner as in Alain's case (Figure 3) but through several steps corresponding to the 6 markers indicated by Michelle. She reported that she had felt more comfortable in 'certain places', namely the shoulder blades, the rib cage, and the wrist, and that she noticed an increase of violin sound in these places. After difficulties in the beginning ('The finger on the string didn't work at all, the wrist helped'), she set the marker for the wrist after about 12 seconds and quickly moved towards a clear and defined sound. When asked if she had used more effort to produce more sound, she stressed that this had happened without any particular effort but with a simultaneous release of tension. And indeed, the sound remained clean and crisp, the increase in volume was not accompanied by stronger bowing noises that would necessarily occur if greater pressure on the string was applied.

In the next stage of the analysis, all participants' interviews were re-examined and content analysed; data treatment was done using ATLAS.ti software (version 8.1, 2017). To 
analyse and compare the large amount of qualitative and quantitative data, methods were tested in an iterative process in an adaption of grounded theory (see Corbin \& Strauss, 2015). Participants' statements, audio recordings, spectral analysis, and sound descriptors were compared, contrasted piece by piece against each other, and reviewed. Examples of tables and diagrams are shown in the Results section.

\section{Results}

In this section, the principal findings will be presented as follows: first, the body parts corresponding to the markers set by the participants; next, the loudness measurements and the interviews; and, finally, the comparison of qualitative and quantitative data.

\section{Markers}

A single body journey (BJ) lasted from 46 to $185 \mathrm{~s}$. Mean values for BJ1 were $84 \mathrm{~s}$, for BJ2 98 s, and for BJ3 110 s. During the three BJs, the 11 participants set 111 markers: 32 in $\mathrm{BJ} 1,43$ in $\mathrm{BJ} 2$, and 36 in $\mathrm{BJ} 3$. In 20 out of $33 \mathrm{BJs}$, they placed 3 or 4 markers, in 8 BJs 2 or fewer markers, and in 5 BJs 5 or more markers. Figure 7 displays the number of markers set by the participants per body part. In BJ1, the knees were marked most often, in BJ2, the rib cage, and in BJ3, the sacral bone. No relationship was found between duration of BJs and numbers of markers. 


\section{Markers and body parts}

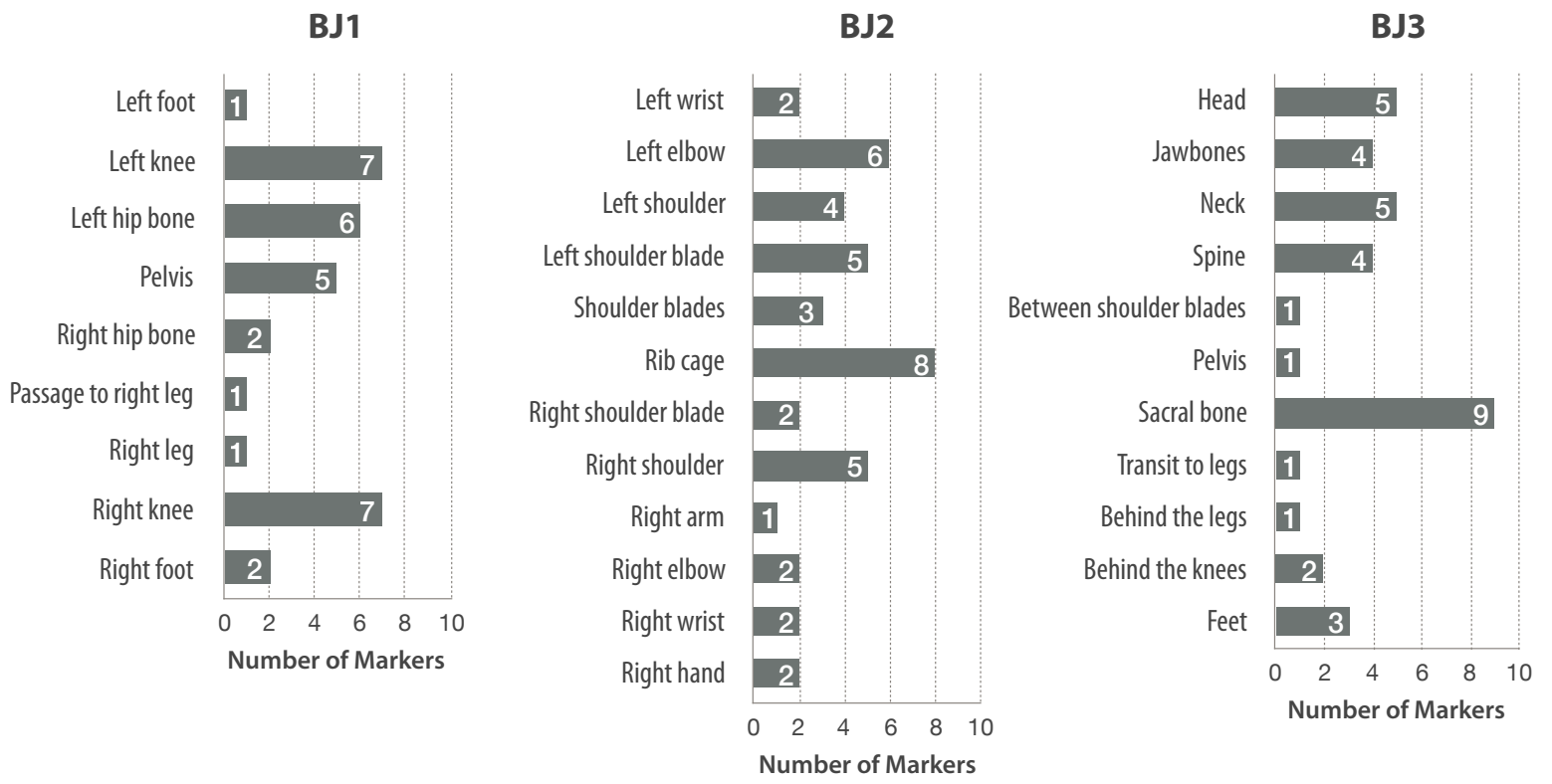

Figure 7. Body parts of the BJs and the corresponding number of markers set by participants.

\section{Loudness}

Minimum and maximum. Table 1 presents the minimal and maximal loudness values of each participant's journeys (examples are shown in Figures 3 and 6) as well as the ratios of the maximal and minimal values. The sone values indicate a stable sound lasting at least 1 second and refer to the tone, without the noise from the bow changes. Doubling the sone values or halving them corresponds to doubling or halving the perceived dynamic sensation.

Table 1. Loudness extremes

\begin{tabular}{|c|c|c|c|c|c|c|c|c|c|c|}
\hline \multirow[b]{2}{*}{ Participant } & \multirow[b]{2}{*}{ Grade } & \multicolumn{3}{|c|}{ BJ1 } & \multicolumn{3}{|c|}{$\mathrm{BJ} 2$} & \multicolumn{3}{|c|}{$\mathrm{BJ} 3$} \\
\hline & & Min & Max & Ratio & Min & Max & Ratio & Min & Max & Ratio \\
\hline Hanna & BA 1 & 3.5 & 5.0 & 1.43 & 3.3 & 5.2 & 1.58 & 3.3 & 5.3 & 1.61 \\
\hline Michelle & BA 3 & 3.2 & 5.8 & 1.81 & 3.1 & 6.1 & 1.97 & 4.6 & 7.1 & 1.54 \\
\hline Marie & BA 3 & 3.1 & 4.5 & 1.45 & 3.1 & 4.3 & 1.39 & 3.1 & 4.5 & 1.45 \\
\hline Maude & BA 3 & 5.5 & 7.8 & 1.42 & 6.5 & 8.7 & 1.34 & 7.1 & 8.4 & 1.18 \\
\hline Rose & BA 3 & 3.6 & 5.4 & 1.50 & 3.2 & 4.6 & 1.44 & 2.8 & 4.0 & 1.43 \\
\hline Jean & MA 1 & 4.4 & 6.2 & 1.41 & 4.8 & 6.2 & 1.29 & 4.3 & 5.8 & 1.35 \\
\hline
\end{tabular}




\begin{tabular}{lllllllllll} 
Alain & MA 1 & 4.4 & 5.4 & 1.23 & 4.4 & 7.2 & 1.64 & 4.8 & 6.5 & 1.35 \\
Laura & MA & 2.8 & 4.2 & 1.50 & 4.0 & 5.2 & 1.30 & 3.5 & 5.0 & 1.43 \\
Emilie & MA 2 & 4.0 & 5.9 & 1.48 & 5.0 & 6.9 & 1.38 & 4.2 & 6.8 & 1.62 \\
Sophie & MA 2 & 2.9 & 4.9 & 1.69 & 4.0 & 6 & 1.50 & 4.0 & 5.9 & 1.48 \\
Julie & MA & 5.9 & 7.1 & 1.20 & 5.8 & 7.9 & 1.36 & 6.7 & 8.5 & 1.27 \\
\hline Mean & & & & 1.47 & & & 1.47 & & & 1.43 \\
\hline
\end{tabular}

Six of the 11 participants reached their maximum loudness in BJ 2, two in BJ3, one in $\mathrm{BJ} 1$, one participant in both $\mathrm{BJ} 1$ and $\mathrm{BJ} 2$, and one participant achieved the same maximum in all three BJs. One participant (Marie) generally played more softly $(\min =3.1$ sones, $\max \leq$ 4.5 sones), and two participants (Maude and Julie) played with more loudness ( $\min \geq 5.5$ sones, $\max \geq 7.1$ sones) than the other eight. Most of the ratios were above 1.4 , which can be considered as a relevant change.

Loudness and body regions. Figure 8 shows the body regions corresponding to the maximum loudness: in BJ1, 4 times the left side (one for passing from left foot to left knee, two for the left knee, and one without a marker), 7 times the pelvic region, and 6 times the right leg (two for the right knee and right foot, and two without a particular marker); in BJ2, once the left side (without marker), 7 times the rib cage region, and 5 times the right side (three for the right hand, and two without a marker); in BJ3, 7 times the head region including jaw bones and neck, 4 times the lower back including the sacral bone, and once the lower legs/feet. Ten times the maximum loudness values were reached twice during the same BJ (six times in $\mathrm{BJ} 1$, three times in $\mathrm{BJ} 2$, and once in $\mathrm{BJ} 3$ ). 


\section{Maximum loudness and body regions}

BJ1

\section{7}

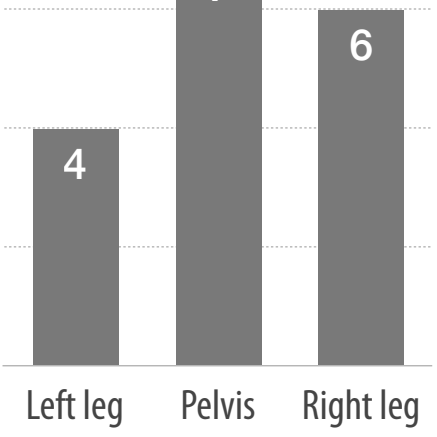

BJ2

7

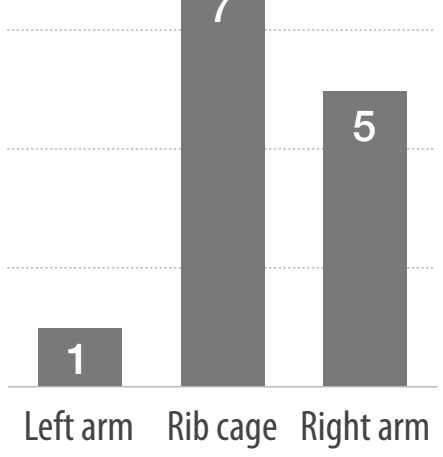

BJ3

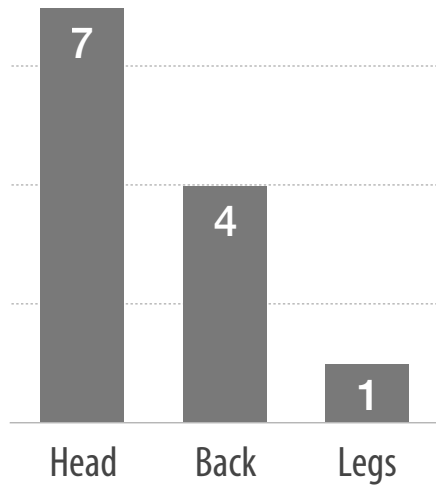

Figure 8. Number of participants per body region who reached maximum loudness values. Note that in $\mathrm{BJ} 1$ and $\mathrm{BJ} 2$, the maximum loudness values were mostly reached in the middle (sacral bone and rib cage) or in the last part of the BJ (right extremities), whereas in BJ3 more than half of the participants reached these values in the head region.

In $\mathrm{BJ} 1$ and $\mathrm{BJ} 2$, correlations were observed regarding laterality in the loudness curves of eight participants. If the loudness increased or decreased on one side of the body in BJ1, this was also the case in BJ2. For example, if an increase occurred in the region of the left knee in $\mathrm{BJ} 1$, there also was an increase in the region of the left elbow in $\mathrm{BJ} 2$; or if a decrease occurred in the region of the right hip bone in $\mathrm{BJ} 1$, there also was a decrease in the region of right shoulder in $\mathrm{BJ} 2$.

\section{Interviews}

Although all participants reported not having had previous experience with the body scan, they coped well with the BJs and the interviews that followed. ${ }^{5}$ During the interviews, participants often took their time between the sentences to recall their body sensations and to find suitable expressions to describe them. They mentioned, for example, 'the transition to the

\footnotetext{
${ }^{5}$ With the exception of Hanna, as mentioned above.
} 
legs was blurry' (Julie) and 'it took me incredibly long to find the hip bones' (Sophie). A total of 149 statements of this kind were collected. In 105 of these, participants reported feelings of ease or unease, which included different grades of physical well-being, stability, easiness, difficulty, relaxation, precision, freedom, and 'groundedness'. Twenty-five statements referred to the sound volume (e.g. Michelle: 'It sounded louder'), 11 to the sound quality (e.g. Marie: 'The sound quality in the right hand was more quiet, more clear'), and 8 statements had a neutral or general meaning (e.g. Jean: 'I felt the arm weight when I moved on'). Furthermore, participants repeatedly made statements related to laterality (14), playing effort (10), time or tempo (9), and/or fluidity of movement (8).

When participants were asked if an extra effort had been necessary to increase the volume, they negated that. They rather indicated a moment of relaxation, an unintended increase of arm weight, or a sensation of greater lightness (e.g. Michelle: 'It relaxed a little bit in the knees', Julie: 'not more effort, but more arm weight', Laura: 'a feeling of more lightness').

\section{Comparison of Qualitative and Quantitative Data}

Interviews and loudness measurement. Twenty-one of the 149 statements collected during the interviews could not be matched with the results of the sound analysis because of missing markers or a neutral or a general meaning. Ninety-two of the remaining 128 statements referred to different grades of ease. We expected that feelings of ease would be reflected in an increase of loudness and sound stability and vice versa. Indeed, in 86 of these statements, the loudness values were higher or more stable when participants reported having felt at ease, or lower or less stable when participants reported having not felt at ease (Table 2). Twenty-five statements referred to the sound volume and were, with one exception, all in line with the measured loudness values. Eleven statements referred to a better sound quality and were reflected in higher loudness values as expected. 
Table 2. Participants'statements relative to loudness values.

\begin{tabular}{|c|c|c|c|c|}
\hline \multirow[b]{2}{*}{ Statements } & \multirow[b]{2}{*}{$\mathrm{n}$} & \multicolumn{3}{|c|}{ Loudness values } \\
\hline & & Expected change & As expected & Different \\
\hline \multicolumn{5}{|l|}{ Grades of ease } \\
\hline $\begin{array}{l}\text { positive (at } \\
\text { ease) }\end{array}$ & 64 & Increase/stability & 59 & 5 \\
\hline $\begin{array}{l}\text { - negative (not } \\
\text { at ease) }\end{array}$ & 28 & Decrease/instability & 27 & 1 \\
\hline Increase of sound & 24 & increase & 23 & 1 \\
\hline Decrease of sound & 1 & decrease & 1 & \\
\hline $\begin{array}{l}\text { Better sound } \\
\text { quality }\end{array}$ & 11 & Increase/stability & 11 & \\
\hline $\begin{array}{l}\text { No corresponding } \\
\text { marker }\end{array}$ & $13^{\mathrm{a}}$ & & & \\
\hline \multirow[t]{2}{*}{ Neutral expression } & $8^{\mathrm{a}}$ & & & \\
\hline & 149 & & 121 & 7 \\
\hline
\end{tabular}

${ }^{a}$ Could not be matched with the results of the sound analysis (i.e. lack of information respectively neutral content)

Subtle sound changes and sound analysis. Finally, seven of the 128 statements that could not be related to the loudness measurement were analysed further to explore a possible connection between body awareness and sonic results. In six cases, the spectral roll-off and the spectral centroid representation together with the spectral analysis highlighted more subtle timbre changes or body sensations to which the participants referred. The most evident one was the roll-off stability (Figure 5) due to a certain body stability reported by participants or the roll-off instability due to a searching phase when the participants were shifting their awareness during the BJ. Indeed, the stationarity of the roll-off corresponded to a body stability and vice versa. In only one case, no relation could be found between the participants subjective experience and sound analysis.

\section{Discussion}

\section{Main Findings}

Table 2 shows the most important finding. In $95 \%$ of the cases, the players' perception of grades of ease and dynamics could be related easily to the loudness measurement. In practical terms, this indicates that non-judgmental, plain awareness of different body regions has a direct impact on the instrumental sound. As expected, the awareness of feelings of well- 
being and ease during the BJs correlated with higher dynamics and sound stability. From the data shown in Table 1, it also can be seen that the ratio between the maximal and minimal loudness value for each participant corresponded to a relevant change of loudness.

The body regions with the highest positive impact on instrumental sound were the pelvis, right leg, rib cage and head (Figure 8). At first, it appears that these body regions are not directly linked to instrumental technique, but our findings indicate that by using body awareness during the BJs, such a link becomes established. In fact, participants regularly stated that volume increases had happened unintentionally and without a specific effort. These increases were accompanied by sensations of increased arm weight, general relaxation, or feelings of lightness. This enables us to hypothesize that body awareness during instrument playing induces non-voluntary changes in the musculoskeletal system that influence the efficiency of the instrumental technique.

\section{Implications for Practice}

The body journeys can be adapted easily for teaching sessions and workshops, enabling instructors and students to create a space for sharing an experience in a context of mutual participation, beyond the need to fulfil externally imposed goals. Tools such as Sonic Visualiser ${ }^{6}$ and Audacity ${ }^{7}$ are readily available at no cost. We consider that regular spectral analysis carried out during the teaching process can be used to clarify the understanding of sonic improvement during the development of instrumental skills.

Based on our results, we assume that body awareness not only has a significant impact on single tones but also on whole musical phrases or passages. BJs could be helpful in discovering individual body spots that might serve as 'anchors' during performances, thus facilitating specific sounds or a particular musical expression, helping to surmount technical

\footnotetext{
${ }^{6}$ Sonic Visualiser: An Open Source Application for Viewing, Analysing, and Annotating Music Audio Files [Computer software]. (2017). Retrieved from https://sonicvisualiser.org

${ }^{7}$ Audacity®: Free Audio Editor and Recorder [Computer software]. (2018). Retrieved from https://audacityteam.org/
} 
difficulties, and shifting one's attention when caught up by stage fright or negative thoughts. Body awareness creates space for exploration and creativity, not only during lessons, but also during practicing, rehearsing, and performing.

\section{Ideas for Future Research}

Taken together, our approach combining BJs with interviews and sound analysis proved to be suitable to detect links between body awareness and instrumental sound. Although the limited number of participants and types of instruments does not allow us to draw more general conclusions, e.g. an extrapolation from string to other types instruments, the first results presented here might stimulate ideas for future research.

In some cases, the reports of the participants were not sufficiently detailed, rendering later comparison of interviews and sound analysis difficult. It therefore is important to encourage the participants to describe their experience during the BJs in great detail. Based on our experience, interviews after the BJs should be held in two parts with space for free narration at the beginning, followed by a semi-structured discussion. A video recording of the interviews could be helpful to record details such as when participants show the body parts to which they refer.

\section{Conclusions}

Body awareness is a straightforward means for acquiring 'self-contact' in an openminded manner. The body always offers the possibility to become aware of the present moment, be it in a professional context or in private life. BJs put the focus on the lived experience; they are easy to carry out and therefore highly suitable for introducing body awareness in a teaching environment. Body awareness during instrument playing has a direct impact on the sound. It can open musicians' sensory perception and encourage the development of self-knowledge and stage presence. Sound analysis additionally allows quantifying the influence of the personal experience on instrumental sound. 
Studies involving other groups and instruments during prolonged periods of time are necessary to further explore the impact of body awareness on instrumental sound and technique, the interplay within an ensemble, and psychological and physical well-being, including stage fright, professional stress, and related health issues.

\section{References}

ATLAS.ti (Version 8.1) [Computer Software]. (2017). Berlin, Germany: Scientific Software Development GmbH. Retrieved from http://www.atlasti.com

AudioSculpt (Version 3.4.5) [Computer Software]. (2016). Paris, France: IRCAM. Retrieved from http://www.ircam.fr/

Bai, H., Beatch, M., Chang, D., \& Cohen, A. (2017). Recalibration of mindfulness for education. In M. Powietrzyńska \& K. Tobin (Eds.), Weaving complementary knowledge systems and mindfulness to educate a literate citizenry for sustainable and healthy lives (pp. 21-40). Rotterdam, Netherlands: SensePublishers. doi:10.1007/978-94-6351-1827_2

Bellinger, D. B., DeCaro, M. S., \& Ralston, P. A. S. (2015). Mindfulness, anxiety, and highstakes mathematics performance in the laboratory and classroom. Consciousness and Cognition, 37, 123-132. doi:10.1016/j.concog.2015.09.001

Bishop, S. R., Lau, M., Shapiro, S., Carlson, L., Anderson, N. D., Carmody, J., \& Devins, G. (2004). Mindfulness: A proposed operational definition. Clinical Psychology: Science and Practice, 11(3), 230-241. doi:10.1093/clipsy.bph077

Buchanan, H. J., \& Hays, T. (2014). The influence of body mapping on student musicians' performance experiences. International Journal of Education \& the Arts, 15(7). Retrieved from http://eric.ed.gov/?id=EJ1039555

Corbin, J. M., \& Strauss, A. L. (2015). Basics of qualitative research: techniques and procedures for developing Grounded Theory (4th ed. revised). Los Angeles, CA: SAGE Publications. 
Czajkowski, A.-M. L., \& Greasley, A. E. (2015). Mindfulness for singers: The effects of a targeted mindfulness course on learning vocal technique. British Journal of Music Education, 32(2), 211-233. doi:10.1017/S0265051715000145

Dreeben, S. J., Mamberg, M., \& Salmon, P. (2013). The MBSR body scan in clinical practice. Mindfulness, 4(4), 394-401. doi:10.1007/s12671-013-0212-z

Gembris, H., Heye, A., \& Seifert, A. (2018). Health problems of orchestral musicians from a life-span perspective: Results of a large-scale study. Music \& Science, 1 , 2059204317739801. doi:10.1177/2059204317739801

Jäncke, L. (2011). Emotionale Belastungen bei Berufsmusikern und ihre Folgen [Negative emotional impact on professional musicians and the consequences]. Zeitschrift für Psychiatrie, Psychologie und Psychotherapie, 59(1), 57-64. doi:10.1024/1661$4747 / \mathrm{a} 000052$

Kabat-Zinn, J. (2012). Mindfulness for beginners: Reclaiming the present moment and your life. Boulder, CO: Sounds.

Kee, Y. H., \& John Wang, C. K. (2008). Relationships between mindfulness, flow dispositions and mental skills adoption: A cluster analytic approach. Psychology of Sport and Exercise, 9(4), 393-411. doi:10.1016/j.psychsport.2007.07.001

Kerr, C. E., Sacchet, M. D., Lazar, S. W., Moore, C. I., \& Jones, S. R. (2013). Mindfulness starts with the body: Somatosensory attention and top-down modulation of cortical alpha rhythms in mindfulness meditation. Frontiers in Human Neuroscience, 7, 12. doi:10.3389/fnhum.2013.00012

Landsman-Dijkstra, J. J. A., Wijck, R. van, Groothoff, J. W., \& Rispens, P. (2004). The shortterm effects of a body awareness program: better self-management of health problems for individuals with chronic a-specific psychosomatic symptoms. Patient Education and Counseling, 55(2), 155-167. doi:10.1016/j.pec.2004.02.014

Leaver, R., Harris, E. C., \& Palmer, K. T. (2011). Musculoskeletal pain in elite professional 
musicians from British symphony orchestras. Occupational Medicine, 61(8), 549-555. doi:10.1093/occmed/kqr129

Lin, P., Chang, J., Zemon, V., \& Midlarsky, E. (2008). Silent illumination: A study on Chan (Zen) meditation, anxiety, and musical performance quality. Psychology of Music, 36(2), 139-155. doi:10.1177/0305735607080840

Ludwig, S. (2002). Elsa Gindler - von ihrem Leben und Wirken. 'Wahrnehmen was wir empfinden' [Elsa Gindler - about her life and her work: 'To perceive what we sense']. \& Haag, M. (Ed.) Hamburg, Germany: Christians.

Mehling, W. E., Wrubel, J., Daubenmier, J. J., Price, C. J., Kerr, C. E., Silow, T., Viranjini, G., \& Stewart, A. L. (2011). Body awareness: A phenomenological inquiry into the common ground of mind-body therapies. Philosophy, Ethics, and Humanities in Medicine: PEHM, 6, 6. doi:10.1186/1747-5341-6-6

Paparo, S. A. (2015). Embodying singing in the choral classroom: A somatic approach to teaching and learning. International Journal of Music Education, 34(4), 488-498. doi:10.1177/0255761415569366

Peeters, G. (2004). A large set of audio features for sound description (similarity and classification) in the CUIDADO project. Paris, France: Ircam Analysis/Synthesis Team. Rodríguez-Carvajal, R., \& Lecuona, O. (2014). Mindfulness and music: A promising subject of an unmapped field. International Journal of Behavioral Research \& Psychology, 2(3), 27-35. doi:10.19070/2332-3000-140006

Rosenzweig, S., Reibel, D. K., Greeson, J. M., Brainard, G. C., \& Hojat, M. (2003). Mindfulness-based stress reduction lowers psychological distress in medical students. Teaching and Learning in Medicine, 15(2), 88-92. doi:10.1207/S15328015TLM1502_03

Steinmetz, A., Scheffer, I., Esmer, E., Delank, K. S., \& Peroz, I. (2015). Frequency, severity and predictors of playing-related musculoskeletal pain in professional orchestral 
musicians in Germany. Clinical Rheumatology, 34(5), 965-973. doi:10.1007/s 10067-013-2470-5

Steyn, B. J., Steyn, M. H., Maree, D. J., \& Panebianco-Warrens, C. (2016). Psychological skills and mindfulness training effects on the psychological wellbeing of undergraduate music students: An exploratory study. Journal of Psychology in Africa, 26(2), 167-171. doi:10.1080/14330237.2016.1163906

Susini, P., Lemaitre, G., \& McAdams, S. (2012). Psychological measurement for sound description and evaluation. In B. Berglund, G. B. Rossi, J. T. Townsend, \& L. R. Pendrill (Eds.), Measurement with Persons: Theory, Methods, and Implementation Areas (pp. 227-253). New York, NY: Psychology Press.

Teasdale, J. D., Segal, Z. V., Williams, J. M. G., Ridgeway, V. A., Soulsby, J. M., \& Lau, M. A. (2000). Prevention of relapse/recurrence in major depression by mindfulness-based cognitive therapy. Journal of Consulting and Clinical Psychology, 68(4), 615-623. doi:10.1037/0022-006X.68.4.615

Tomasino, B., \& Fabbro, F. (2016). Increases in the right dorsolateral prefrontal cortex and decreases the rostral prefrontal cortex activation after- 8 weeks of focused attention based mindfulness meditation. Brain and Cognition, 102, 46-54. doi:10.1016/j.bandc.2015.12.004

Ussher, M., Spatz, A., Copland, C., Nicolaou, A., Cargill, A., Amini-Tabrizi, N., \& McCracken, L. M. (2014). Immediate effects of a brief mindfulness-based body scan on patients with chronic pain. Journal of Behavioral Medicine, 37(1), 127-134. doi:10.1007/s10865-012-9466-5

Weaver, J. O. (2015). The influence of Elsa Gindler. In G. Marlock, H. Weiss, C. Young, \& M. Soth (Eds.), The Handbook of body psychotherapy and somatic psychology (pp. 4046). Berkeley, CA: North Atlantic Books.

Zautra, A. J., Davis, M. C., Reich, J. W., Nicassario, P., Tennen, H., Finan, P., \& Irwin, M. R. 
(2008). Comparison of cognitive behavioral and mindfulness meditation interventions on adaptation to rheumatoid arthritis for patients with and without history of recurrent depression. Journal of Consulting and Clinical Psychology, 76(3), 408-421. doi:10.1037/0022-006X.76.3.408

Zhang, C.-Q., Si, G., Duan, Y., Lyu, Y., Keatley, D. A., \& Chan, D. K. C. (2016). The effects of mindfulness training on beginners' skill acquisition in dart throwing: A randomized controlled trial. Psychology of Sport and Exercise, 22, 279-285. doi:10.1016/j.psychsport.2015.09.005

Zylowska, L., Ackerman, D. L., Yang, M. H., Futrell, J. L., Horton, N. L., Hale, T. S., \& Smalley, S. L. (2007). Mindfulness meditation training in adults and adolescents with ADHD: A Feasibility Study. Journal of Attention Disorders, 11(6), 737-746.

doi:10.1177/1087054707308502 